\title{
Overview and Algorithmic Approach to Management of Male and Female Pattern Hair Loss
}

\author{
Mayank Singh ${ }^{1}$ Aparna Acharya ${ }^{2}$ \\ ${ }^{1}$ Radiance Cosmedic Centre, New Delhi, India \\ 2 Hair Plus, Pune, Maharashtra, India \\ Indian J Plast Surg 2021;54:416-421.
}

\begin{abstract}
Address for correspondence Mayank Singh, MS, MCh (Plastic Surgery), Radiance Cosmedic Centre, Greater Kailash and Janakpuri, New Delhi, India (e-mail: drmayanksingh@yahoo.in).
\end{abstract}
Abstract
Keywords
- hair transplant
- algorithm of treatment
- pattern hair loss
- donor area
- planning of hair transplant

Male and female pattern hair loss (PHL) is an innocuous condition, but it has a major psychological impact on the sufferer. This paper aims to provide a simple algorithmic approach toward diagnosis, staging, and treatment of PHL in males and females. It also aims at simplifying the decision-making process for the surgeon with regard to timing and extent of procedure for hair transplant surgeries. Various treatment options, their merits and demerits, along with scientific evidence supporting or not supporting the treatment options are discussed in detail.

\section{Introduction}

Male and female pattern hair loss (PHL), also known as androgenetic alopecia (AGA), is a common disorder affecting both sexes, although its incidence is greater in males than females. ${ }^{1}$

The current market-driven scenario is exploiting PHL as a commodity rather than a clinical condition. Hence, it is important that we, as treating doctors, stay firm on our sound clinical judgement and treat this entity judiciously in a scientific manner. ${ }^{2}$

For treating PHL correctly, it is imperative to rule out other conditions mimicking it first. Once the correct diagnosis is established, we need to choose an appropriate treatment, depending upon the stage of baldness, ages of the individua$\mathrm{ls}$, their expectations from the treatment, and our ability to fulfill these expectations in the long run. Since multiple therapeutic options are available for the treatment of AGA, we need to know which treatments are evidence-based. While considering surgical treatment, the planning of surgery, its timing with regard to the stage of baldness, age, donor area conditions, etc. are as important as the actual surgical technique.

\section{Algorithm for Diagnosing Pattern Hair Loss (-Fig. 1) \\ History}

Age at onset-PHL usually starts around the second or third decade of life.

Duration in years-It is prolonged in case of PHL and nonpattern baldness often has a short history.

Speed of progression-It is slow in pattern baldness.

Family history of baldness-often positive in pattern baldness.

Treatment history including salon treatment for hair straightening, perming, bleaching, and others that can induce telogen effluvium.

Concomitant diseases like thyroid or other endocrine disorders. published online

December 14, 2021
DOI https://doi.org/ $10.1055 / \mathrm{s}-0041-1739256$ ISSN 0970-0358.

\footnotetext{
(c) 2021. Association of Plastic Surgeons of India. All rights reserved. This is an open access article published by Thieme under the terms of the Creative Commons Attribution-NonDerivative-NonCommercial-License, permitting copying and reproduction so long as the original work is given appropriate credit. Contents may not be used for commercial purposes, or adapted, remixed, transformed or built upon. (https://creativecommons.org/ licenses/by-nc-nd/4.0/)

Thieme Medical and Scientific Publishers Pvt. Ltd., A-12, 2nd Floor, Sector 2, Noida-201301 UP, India
} 
Psychiatric disorders if any-trichotillomania leads to patchy hair loss. Various antidepressants can be the reason for gradual hair thinning.

Medication history for other conditions-Various drugs can induce diffuse hair loss.

History of consumption of drugs and supplements for bodybuilding or sports-Excessive amounts of protein ingestion, consumption of anabolic steroids, and similar drugs often induce hair loss.

Smoking, alcoholism.

Menstrual and obstetric history as well as polycystic ovarian disease in females.

Recent dietary changes-Drastic reduction in calory intake, complete stoppage of carbohydrates and fats, and similar fad diets have detrimental effects on hair health.

\section{Clinical Examination}

This includes a general assessment to rule out anemia, any obvious systemic illness, or endocrine diseases, followed by examination of hair and scalp.

Assessing distribution of thinning and bald areas and determining whether it fits into pattern baldness, that is, affecting specific areas, or diffuse thinning.

Stage of pattern baldness

Scalp skin condition, whether normal or abnormal. Shiny, scaly, scarred, and erythematous skin is frequently seen in scarring alopecias.

\section{Hair Caliber and Density}

Assessment of potential donor area

Particular hairstyles inviting traction alopecia.

Pull Test-It is usually negative in PHL, whereas it is positive in telogen effluvium.

\section{Trichoscopy}

Trichoscopy is an important tool in the diagnosis of pattern alopecia. The permanent zone remains unaffected in the pattern alopecia. The nonpermanent_zone shows anisotrichosis, varying diameters of hair shafts with peripilar halo.

Scarring alopecia show signs of inflammation, that is, erythema, scaling and hyperkeratosis, seborrhea, and signs of scarring, that is, atrophy and loss of hair follicle ostia.

Telogen effluvium is characterized by findings like decreased hair density with the presence of empty follicles. It can be easily differentiated from PHL due to the absence of hair shaft diameter variation and peripilar halo. It affects the entire scalp unlike PHL where the donor area is not affected. Telogen effluvium is the diagnosis of exclusion on trichoscopy. When entire scalp, including permanent zone, shows, anisotrichosis or diagnosis of diffused unpatterned alopecia (DUPA) is established.

\section{Investigations}

Usually, laboratory tests are not needed to establish the diagnosis of PHL, but if accompanying telogen effluvium is suspected, hemogram, serum ferritin levels, and other appropriate tests might help in planning the treatment. In females, thyroid function tests, serum testosterone and prolactin levels are recommended along with screening to rule out polycystic ovarian disease. ${ }^{3}$

Scalp biopsy: It is only needed to establish the diagnosis of nonpattern alopecia especially scarring alopecia and is not routinely recommended.

After the diagnosis of male pattern baldness is established, appropriate treatment should be advised.

\section{Algorithm for Treatment of Male Pattern Baldness (-Fig. 2)}

Treatment should be based on current Norwood grade of baldness, age, and family history of baldness. One should be very cautious while treating patients below 25 years of age. Donor area quality and facial framing, whether lost or preserved, plays a major role in decision-making.

\section{Algorithm for Treatment of Female Pattern Baldness (-Fig. 3)}

Ludwig staging, donor area quality, concurrent illnesses, and expectations out of treatment play vital roles in the decisionmaking of female pattern baldness treatment.

\section{- Topical minoxidil solution}

It is administered at a dosage of $1 \mathrm{~mL}$, twice daily. It prolongs the anagen phase, induces vasodilatation, and increases hair shaft diameter. The efficacy of minoxidil solution varies in different studies, but it is well-established that it is safe and induces a significant increase in hair count. ${ }^{4}$ \% minoxidil is more effective than the $2 \%$ or $3 \%$ solution. Minoxidil foam is also available and sometimes it is better tolerated for people who are allergic to the polyglycol vehicle of minoxidil solution. ${ }^{5}$

Patients should be warned that in the initial 2 to 8 weeks, a temporary telogen effluvium may occur in some, which is self-limiting and subsides when subsequent anagen regrowth begins, and it should not be a cause for treatment cessation. $^{5}$

Treatment should be continued indefinitely, as termination of medication results in hair shedding within 3 to 4 months. As a safety measure, minoxidil should be avoided during pregnancy and lactation. ${ }^{6}$ Adverse reactions are rashes, itching, scalp irritation, and mild headache, which are usually self-limiting with supportive treatment. Very rarely, there are severe allergic reactions that necessitate stoppage of application and antiallergic treatment.

\section{- Oral 5- $\alpha$-reductase-inhibitors}

\section{Finasteride and Dutasteride}

Finasteride $1 \mathrm{mg}$ per day is approved by United States Food and Drug Administration (FDA) for hair restoration purposes. Finasteride is not indicated in women and is contraindicated in pregnant women because of the risk of feminization of a male fetus. Finasteride-treated men must avoid donating 


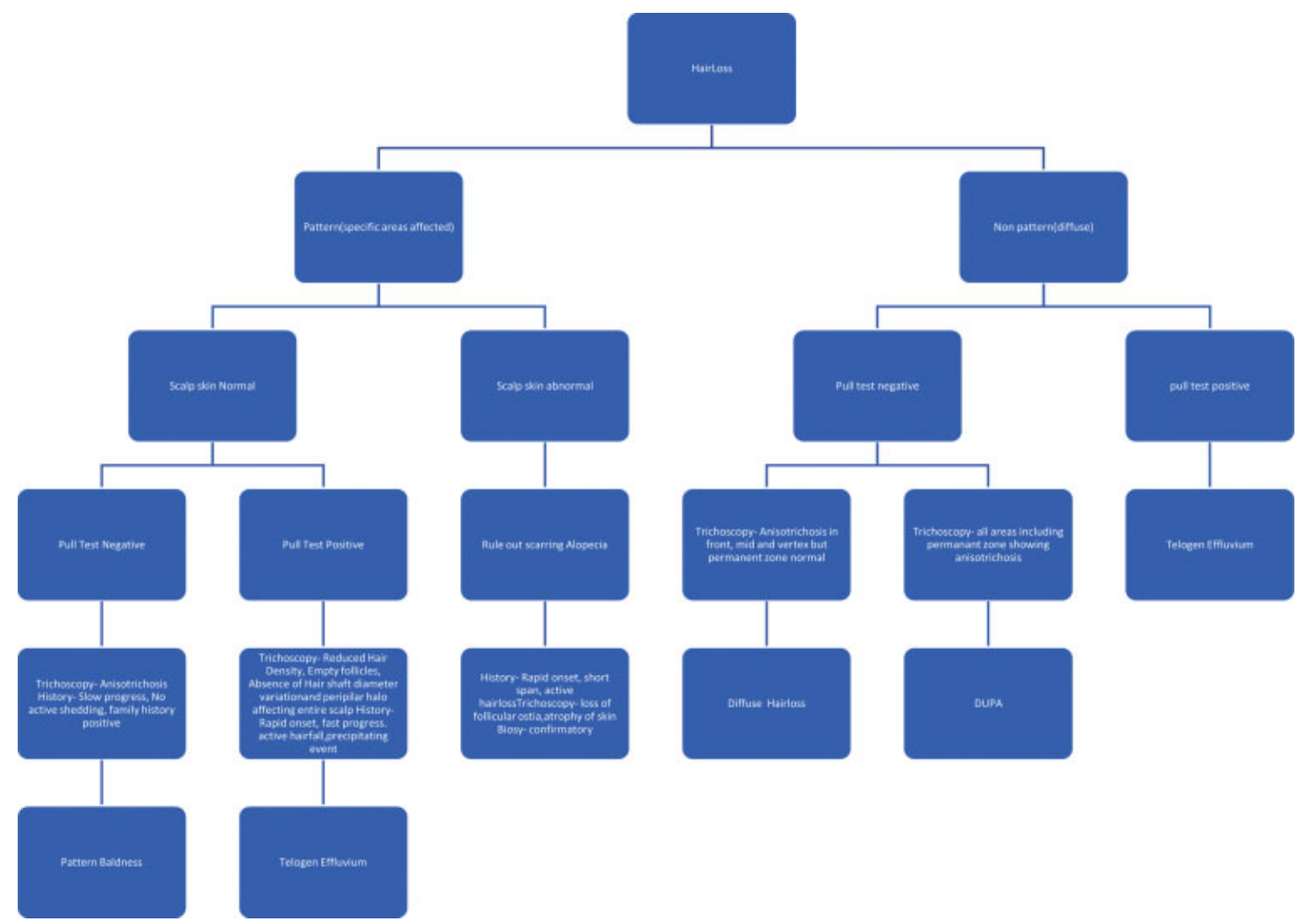

Fig. 1 Algorithm for diagnosis of hairloss.

blood. Patients should be aware of the reduction of prostatespecific antigen with long-term finasteride treatment. PSA is an important indicator in prostate cancer screening in men older than 45 years. ${ }^{4}$

The treatment with these drugs has to be continued for a minimum of 8 to 12 months for visible results. Some studies indicate that these drugs, especially finasteride, are effective even in low doses, so maintenance therapy can be continued further in lower dosage if needed.

Dutasteride is not approved by US FDA but is used offlabel frequently for hair restoration purpose in a dose of $0.5 \mathrm{mg}$ per day. Both these drugs have been proven to have a good therapeutic effect in terms of increased hair counts. Dutasteride seems to provide a better efficacy compared with finasteride, and the two drugs appear to show similar rates of adverse reactions, especially with respect to sexual dysfunction. Further clinical studies are needed to compare the two drugs in terms of efficacy and side effects. ${ }^{4}$

Both the drugs have sexual side effects of decreased libido, premature ejaculation, and erectile dysfunction. The incidence of these side effects varies with different studies. Some studies indicate that the side effects of finasteride are completely reversible after stoppage of medicine but the same is not true with dutasteride.

There is another entity called psychological sexual dysfunction ${ }^{7}$ after starting these medicines, as there is extensive misinformation about these drugs on Internet, and patients tend to start experiencing it after reading about it.
Hence, it is necessary to discuss sexual dysfunction side effects of these drugs with patients before starting the treatment, and it is better to avoid these drugs in people who are to be married soon or planning to start a family and those who already have sexual dysfunction or are apprehensive about it. ${ }^{7}$

\section{Oral minoxidil}

Minoxidil $2.5 \mathrm{mg}$ orally once a day in female patients with PHL, and for males who cannot tolerate topical minoxidil, it shows preferable outcomes in the form of decreased hair shedding and improved hair density. The mean severity score reduces to 2.3 at 6 months and 2.6 at 12 months. ${ }^{8}$ Mild adverse effects including urticaria, postural hypotension, and facial hypertrichosis are reported. However, there are reported cases of precipitation of chest pain, and a baseline cardiac evaluation is recommended before starting oral minoxidil, especially for those above 35 years of age and with a positive family or self-history of cardiac events.

\section{Nutritional therapy}

Various nutritional supplements are advised in PHL like B complex (especially vitamin B12), vitamin D3, omega 3 and omega 6 , folic acid, vitamin E, iron, calcium, selenium, manganese, zinc, copper, other trace elements like vitamin C and vitamin A, etc. ${ }^{9,10}$ Multiple studies have shown the beneficial effects of nutritional supplements in the treatment of PHL with or without concomitant finasteride. ${ }^{9,10}$ 


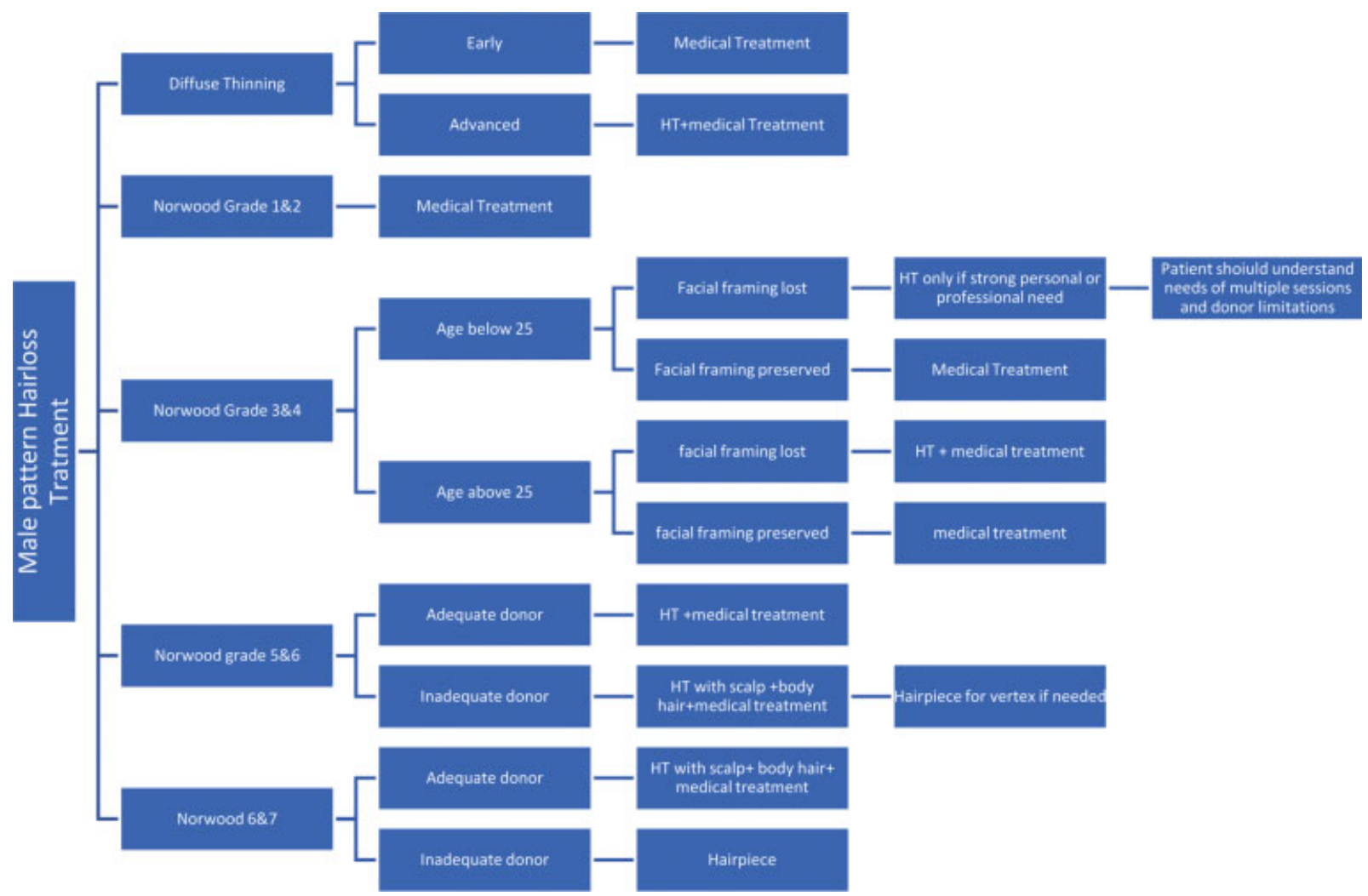

Fig. 2 Algorithm for male pattern hairloss treatment.

Further studies are needed to establish it as a standard treatment.

\section{Other topical agents}

Numerous isolated studies have propagated various topical agents claimed to be beneficial in PHL. These are caffeine,

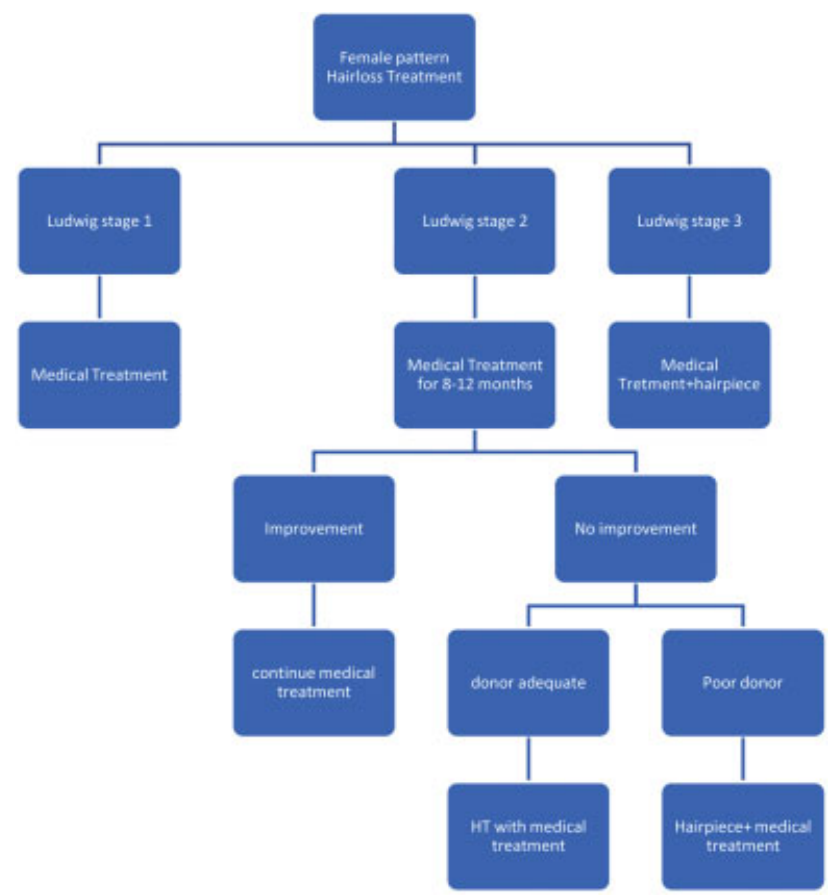

Fig. 3 Algorithm for treatment of female pattern hairloss. amenexil, saw palmetto, curcumin, topical finasteride, ketoconazole, tea tree oil, hibiscus extracts, various other herbal extracts, etc. However, conclusive studies are missing. ${ }^{11-13}$

\section{Platelet-rich plasma}

Platelet-rich plasma (PRP) injections for local hair restoration in patients with AGA seem to increase hair number and thickness with minimal or no collateral effects. However, the current evidence does not support this treatment modality ${ }^{14}$ over other treatments due to the lack of clinical evidence, established protocols (i.e., number of sessions, centrifugation, zones to be injected, etc.), and long-term follow-up outcomes.

The addition of PRP might be useful in improving the outcomes of hair transplantation procedures, but there is no evidence whether PRP is more effective than minoxidil or finasteride treatments. ${ }^{14}$

\section{Low-level laser treatment}

Low-level laser treatment (LLLT) is a safe and effective tool for treating PHL, but more studies with focus on clinical improvements, comparison of diverse laser settings, and treatment regimens will be helpful in developing a standardized treatment regimen. ${ }^{15}$

\section{Combination therapies}

Various treatment modalities can be combined to give better outcomes. The only clinically proven combination is minoxidil with finasteride. ${ }^{16,17}$ 
Multiple small studies evaluate combining minoxidil with nutritional therapy, PRP, or other modalities of treatment. However, large and controlled studies are lacking. ${ }^{18-23}$

\section{Hair Transplant}

Despite advances in medical therapy, hair transplantation remains the only means of permanent hair restoration in severe AGA. There are certain facts which need to be always taken into account while subjecting any person to hair transplant.

- Every person who has started getting bald has the potential to reach grade 7 baldness. There are no absolute predictors about the time frame within which it will happen.

- Permeant donor zone of the scalp is limited. If we want to leave behind an aesthetically acceptable donor area, we can harvest a maximum of 6000 follicular units (FU) from an average donor by follicular unit extraction (FUE) technique and approximately 1000 more FU if we use the follicular unit transplantation (FUT) technique. It is clear by following mathematical calculation. ${ }^{24,25}$

Total scalp hair bearing area is approximately 500 sq. $\mathrm{cm}$ with an average density of $1 \mathrm{FU} / \mathrm{sq}$. $\mathrm{mm}$. That gives a total hair count of approximately 1,00,000. Only 25 to 30 percent of scalp is part of the safe donor zone. That gives us approximately 25000 donor hair. To maintain donor aesthetics, we need to leave behind at least 50 percent of hair. It translates to approximately 12500 hair and approximately $6000 \mathrm{FU}$ in an average donor. 24,25

- In grade 7 baldness, the area to be covered is around 300 to $350 \mathrm{~cm}^{2}$

- Even though transplant is a camouflage technique and a density of approximately 30 to $40 \mathrm{FU} /$ gives adequate aesthetic result, we still need approximately 9000 to $10000 \mathrm{FU}$ to be able to provide complete coverage in grade 7 baldness. $^{26}$

That gives us a deficit of approximately 3000 to $4000 \mathrm{FU}$. Except for beard hair in some people, not everyone has adequate body hair to be able to give the desired number of grafts to cover the bald area.

- Vertex hair transplant is not advised for patients below 35 years of age, as vertex keeps expanding till that age. It can create a halo around the transplanted area with progressive balding, giving a very unnatural appearance.

- We should not fall prey to patients' unrealistic expectations from hair transplant and should be able to counsel them properly about when to undergo hair transplant and with what coverage and density.

\section{Guidelines for Patient Selection in Hair Transplant}

- Hair transplant surgeons must be very cautious while treating young people (i.e., below 25 years of age). If they are between grade 1 to 3 , medical treatment is the treatment of choice. Hair transplant should never be done for these patients to lower the hairlines, as this will invariably result in aesthetically unacceptable hairlines with front, mid, and vertex baldness in advanced age.

- Hair transplant is to be approached with caution in diffuse thinning pattern. These patients should always be subjected to medical line of treatment first for, at least, 9 to 12 months before planning HT. Proper counselling is necessary for such patients, as they are likely to lose existing scalp hair over time. This can result in getting back to the same pretransplant density even after the procedure.

- For grade 4 to 5 , hairlines must never be unduly lowered, or very high density given, because donor supply is abundant at the time. These patients can reach advanced grades of baldness, and they should have reserve donor to be able to give coverage later.

- Care should be taken not to damage permanent donor zone by overharvesting or close harvesting. Similarly, one must be careful not to overstep the safe donor zone.

- Hair transplant should be deferred for all people who are in the active and rapidly progressive hair loss phase. In such cases, the primary line of management is medical treatment till the speed of hair loss stabilizes.

In females, hair transplant is to be approached with caution. Every female patient should be investigated to find out whether there is any underlying cause. Hair transplant is to be considered only for grade 2 and 3 and only after the medical line of treatment given for adequate time fails to give results.

\section{Conclusion}

Male and female pattern baldness is a challenging condition to treat. It needs long-term commitment from both the patient and the doctor. It is very important to evaluate patients thoroughly, give adequate time for counselling, and not give in to the patient's unrealistic expectations from treatment or hair transplant.

Long-term planning for donor area management is an integral part of hair transplant surgeries.

We, as medical fraternity, should stand firmly by our scientific knowledge and carry on ethical work, so that clinically proven treatments and hair transplant procedures are not tarnished.

Conflict of Interest

None declared.

\section{References}

1 Kaliyadan F, Nambiar A, Vijayaraghavan S. Androgenetic alopecia: an update. Indian J Dermatol Venereol Leprol 2013;79(05): 613-625

2 Medical WG, Ethics P. Spotlight on medical ethics. Hair Transplant Forum Int 2020;30(06):230-231

3 Birch MP, Messenger JF, Messenger AG. Hair density, hair diameter and the prevalence of female pattern hair loss. Br J Dermatol $2001 ; 144(02): 297-304$ 
4 Blumeyer A, Tosti A, Messenger A, et al; European Dermatology Forum (EDF) Evidence-based (S3) guideline for the treatment of androgenetic alopecia in women and in men. J Dtsch Dermatol Ges 2011;9(Suppl 6):S1-S57

5 Suchonwanit P, Thammarucha S, Leerunyakul K. Minoxidil and its use in hair disorders: a review. Drug Des Devel Ther 2019; 13:2777-2786

6 Goren A, Shapiro J, Roberts J, et al. Clinical utility and validity of minoxidil response testing in androgenetic alopecia. Dermatol Ther (Heidelb) 2015;28(01):13-16

7 Mysore V. Finasteride and sexual side effects. Indian Dermatol Online J 2012;3(01):62-65

8 Randolph M, Tosti A. Oral minoxidil treatment for hair loss: a review of efficacy and safety. J Am Acad Dermatol 2021;84(03): 737-746

9 Rajput RJ. Controversy: is there a role for adjuvants in the management of male pattern hair loss? J Cutan Aesthet Surg 2010;3(02):82-86

10 Beer C, Wood S, Veghte RH. A clinical trial to investigate the effect of Cynatine HNS on hair and nail parameters. ScientificWorldJournal 2014;2014:641723

11 Ashique S, Sandhu NK, Haque SN, Koley K. A systemic review on topical marketed formulations, natural products, and oral supplements to prevent androgenic alopecia: a review. Nat Prod Bioprospect 2020;10(06):345-365

12 Dhariwala MY, Ravikumar P. An overview of herbal alternatives in androgenetic alopecia. J Cosmet Dermatol 2019;18(04):966-975

13 Lee SW, Juhasz M, Mobasher P, Ekelem C, Mesinkovska NA. A systematic review of topical finasteride in the treatment of androgenetic alopecia in men and women. J Drugs Dermatol 2018;17(04):457-463

14 Giordano S, Romeo M, Lankinen P. Platelet-rich plasma for androgenetic alopecia: Does it work? Evidence from meta analysis. J Cosmet Dermatol 2017;16(03):374-381

15 Egger A, Resnik SR, Aickara D, et al. Examining the safety and efficacy of low-level laser therapy for male and female pattern hair loss: a review of the literature. Skin Appendage Disord 2020; 6(05):259-267

16 Chen L, Zhang J, Wang L, Wang H, Chen B. The efficacy and safety of finasteride combined with topical minoxidil for androgenetic alopecia: a systematic review and meta-analysis. Aesthetic Plast Surg 2020;44(03):962-970

17 Gupta AK, Cole J, Deutsch DP, et al. Platelet-rich plasma as a treatment for androgenetic alopecia. Dermatol Surg 2019;45(10): $1262-1273$

18 Suchonwanit P, lamsumang W, Rojhirunsakool S. Efficacy of topical combination of $0.25 \%$ finasteride and $3 \%$ minoxidil versus $3 \%$ minoxidil solution in female pattern hair loss: a randomized, double-blind, controlled study. Am J Clin Dermatol 2019;20(01): 147-153

19 Zhou Y, Chen C, Qu Q et al. The effectiveness of combination therapies for androgenetic alopecia: A systematic review and meta-analysis. Dermatol Ther (Heidelb) 2020;33(04):e13741

20 Kelly Y, Blanco A, Tosti A. Androgenetic alopecia: an update of treatment options. Drugs 2016;76(14):1349-1364

$21 \mathrm{Hu} \mathrm{R}, \mathrm{Xu} \mathrm{F}$, Sheng Y, et al. Combined treatment with oral finasteride and topical minoxidil in male androgenetic alopecia: a randomized and comparative study in Chinese patients. Dermatol Ther (Heidelb) 2015;28(05):303-308

22 Tanglertsampan C. Efficacy and safety of 3\% minoxidil versus combined $3 \%$ minoxidil / $0.1 \%$ finasteride in male pattern hair loss: a randomized, double-blind, comparative study. J Med Assoc Thai 2012;95(10):1312-1316

23 Khandpur S, Suman M, Reddy BS. Comparative efficacy of various treatment regimens for androgenetic alopecia in men. J Dermatol 2002;29(08):489-498

24 Bernstein RM, Rassman WR. The aesthetics of follicular transplantation. Dermatol Surg 1997;23(09):785-799

25 Bernstein RM, Rassman WR. The logic of follicular unit transplantation. Dermatol Clin 1999;17(02):277-295, viii, discussion 296

26 Venkataram A, Mysore V. Logic of hair transplantation. J Cutan Aesthet Surg 2018;11(04):169-172 